\title{
Lattice-based integration algorithms: Kronecker sequences and rank-1 lattices
}

\author{
Josef Dick $^{1}$ - Friedrich Pillichshammer ${ }^{2}$. \\ Kosuke Suzuki $^{3}$ - Mario Ullrich ${ }^{4}$. Takehito Yoshiki ${ }^{5}$
}

Received: 22 February 2017 / Accepted: 4 May 2017 / Published online: 19 May 2017

(C) The Author(s) 2017. This article is an open access publication

\begin{abstract}
We prove upper bounds on the order of convergence of lattice-based algorithms for numerical integration in function spaces of dominating mixed smoothness on the unit cube with homogeneous boundary condition. More precisely, we study worst-case integration errors for Besov spaces of dominating mixed smoothness $\stackrel{\mathbf{B}}{p, \theta}_{p, \theta}$, which also comprise the concept of Sobolev spaces of dominating mixed smoothness $\stackrel{\circ}{ }_{p}^{s}$ as special cases. The considered algorithms are quasi-Monte Carlo rules with underlying nodes from $T_{N}\left(\mathbb{Z}^{d}\right) \cap[0,1)^{d}$,
\end{abstract}

The research of J. Dick, K. Suzuki and T. Yoshiki was supported under the Australian Research Councils Discovery Projects funding scheme (Project Number DP150101770). F. Pillichshammer was supported by the Austrian Science Fund (FWF): Projects F5509-N26, which is part of the Special Research Program "Quasi-Monte Carlo Methods: Theory and Applications".

$凶 \quad$ Friedrich Pillichshammer

friedrich.pillichshammer@jku.at

Josef Dick

josef.dick@unsw.edu.au

Kosuke Suzuki

kosuke-suzuki@hiroshima-u.ac.jp

Mario Ullrich

mario.ullrich@jku.at

Takehito Yoshiki

yoshiki.takehito.47x@st.kyoto-u.ac.jp

1 School of Mathematics and Statistics, The University of New South Wales, Sydney, NSW 2052, Australia

2 Institut für Finanzmathematik und angewandte Zahlentheorie, Johannes Kepler Universität Linz, Altenbergerstraße 69, 4040 Linz, Austria

3 Hiroshima University, Higashihiroshima, Japan

4 Institut für Analysis, Johannes Kepler Universität Linz, Altenbergerstraße 69, 4040 Linz, Austria

5 Department of Applied Mathematics and Physics, Graduate School of Informatics, Kyoto University, Kyoto 606-8561, Japan 
where $T_{N}$ is a real invertible generator matrix of size $d$. For such rules, the worst-case error can be bounded in terms of the Zaremba index of the lattice $\mathbb{X}_{N}=T_{N}\left(\mathbb{Z}^{d}\right)$. We apply this result to Kronecker lattices and to rank-1 lattice point sets, which both lead to optimal error bounds up to $\log N$-factors for arbitrary smoothness $s$. The advantage of Kronecker lattices and classical lattice point sets is that the run-time of algorithms generating these point sets is very short.

Keywords Numerical integration · Quasi-Monte Carlo · Kronecker lattice · Rank-1 lattice points $\cdot$ Zaremba index $\cdot$ Besov space

Mathematics Subject Classification 65D30 - 65D32 - 11K31

\section{Introduction}

In this paper, we study numerical integration of smooth functions on the $d$-dimensional unit cube which satisfy homogeneous boundary conditions. More precisely, we consider Besov spaces of dominating mixed smoothness $\dot{\mathbf{B}}_{p, \theta}^{s}$ which also comprise the concept of Sobolev spaces of dominating mixed smoothness $\stackrel{\circ}{\mathbf{H}}_{p}^{s}$ as special cases. The exact definition of these spaces requires some preparation and will be given in Sect. 2.2. For the moment we just note that the parameter $s$ denotes the underlying smoothness of the functions and that for $f \in \stackrel{\stackrel{B}{B}}{p, \theta}_{p, \theta}$ we have $\operatorname{supp}(f) \subset[0,1]^{d}$.

We study numerical integration

$$
I_{d}(f)=\int_{[0,1]^{d}} f(\boldsymbol{x}) \mathrm{d} \boldsymbol{x} \quad \text { for } f \in \stackrel{\mathrm{B}}{p, \theta}_{p}^{s}
$$

with linear algorithms of the form

$$
Q_{N, d}(f)=\sum_{j=0}^{N-1} a_{j} f\left(x_{j}\right)
$$

for given sample points $\boldsymbol{x}_{0}, \ldots, \boldsymbol{x}_{N-1} \in[0,1]^{d}$ and real weights $a_{0}, \ldots, a_{N-1}$. For the special choice $a_{j}=1 / N$ for all $j=0, \ldots, N-1$, we speak of quasi-Monte Carlo (QMC) algorithms.

The worst-case error of an algorithm $Q_{N, d}$ is the worst absolute integration error of $Q_{N, d}$ over the unit-ball of $\stackrel{\circ}{\mathbf{B}}_{p, \theta}^{s}$, i.e.,

$$
\operatorname{wce}\left(Q_{N, d}, \stackrel{\circ}{\mathbf{B}}_{p, \theta}^{s}\right)=\sup _{\|f\|_{\stackrel{\mathbf{B}}{p}_{p, \theta}^{s}} \leq 1}\left|I_{d}(f)-Q_{N, d}(f)\right|,
$$

where $\|\cdot\|_{\dot{\mathbf{B}}_{p, \theta}^{s}}$ denotes the norm in $\stackrel{\circ}{\mathbf{B}}_{p, \theta}^{s}$.

As integration rules we use what we call general lattice rules which are of the form

$$
Q_{T}(f)=|\operatorname{det}(T)| \sum_{x \in T\left(\mathbb{Z}^{d}\right) \cap[0,1)^{d}} f(\boldsymbol{x})
$$

with an invertible matrix $T \in \mathbb{R}^{d \times d}$. Note that

$$
\mathbb{X}=T\left(\mathbb{Z}^{d}\right)=\left\{T \boldsymbol{x}: \boldsymbol{x} \in \mathbb{Z}^{d}\right\}
$$


is a $d$-dimensional lattice in $\mathbb{R}^{d}$, i.e., a discrete additive subgroup of $\mathbb{R}^{d}$ not contained in a proper linear subspace of $\mathbb{R}^{d}$.

A specific example of such a rule is Frolov's cubature formula introduced in [1] (see also [2] and the survey paper by Temlyakov [3, Section 4.3]). Here a suitable generating matrix $T$ for the underlying lattice $\mathbb{X}$ is found as follows: Define the polynomial $p_{d} \in \mathbb{R}[x]$, $p_{d}(x)=-1+\prod_{j=1}^{d}(x-2 j+1)$. Then $p_{d}$ has only integer coefficients, it is irreducible over the rationals and has $d$ different real roots, say $\xi_{1}, \ldots, \xi_{d} \in \mathbb{R}$. With these roots define the $d \times d$ Vandermonde matrix $B$ by

$$
B=\left(B_{i, j}\right)_{i, j=1}^{d}=\left(\xi_{i}^{j-1}\right)_{i, j=1}^{d} .
$$

Then the generator of the lattice $\mathbb{X}$ used in Frolov's cubature formula is

$$
T=\frac{1}{a}\left(B^{\top}\right)^{-1},
$$

where $a>1$ is a shrinking factor.

It was shown by Dubinin [4], see also [5] and [6], that Frolov's cubature formula achieves the optimal convergence rate for the worst-case error in $\stackrel{\mathrm{B}}{p, \theta}_{p, \theta}^{s}$, which is

$$
\operatorname{wce}\left(Q_{T}, \stackrel{\circ}{\mathbf{B}}_{p, \theta}^{s}\right) \asymp \frac{(\log N)^{(d-1)(1-1 / \theta)}}{N^{s}},
$$

where $N$ is the number of elements of $T\left(\mathbb{Z}^{d}\right)$ that belong to the unit cube $[0,1]^{d}$. The lower bound for these spaces was proven in [2]. See also [7] for techniques to transfer such results to spaces without (or with periodic) boundary conditions. For the Sobolev(-Hilbert) spaces $\stackrel{\circ}{\mathbf{H}}_{2}^{s}=\stackrel{\circ}{\mathbf{B}}_{2,2}^{s}$ the result reads

$$
\operatorname{wce}\left(Q_{T}, \stackrel{\circ}{\mathbf{H}}_{2}^{S}\right) \asymp \frac{(\log N)^{(d-1) / 2}}{N^{s}} .
$$

The problem with Frolov's cubature formula is that one needs to determine which points from the shrunk lattice belong to the unit cube $[0,1]^{d}$. This is in general a very difficult task, especially when the dimension $d$ is large. ${ }^{1}$

It is the aim of this paper to find other general lattice rules whose lattice points are faster to generate on a computer also for large dimensions $d$ and which also achieve optimal convergence rate for the worst-case error with respect to the smoothness $s$, up to $\log N$-terms.

We will prove a very general estimate for the worst-case error in terms of the Zaremba index of the lattice $\mathbb{X}=T\left(\mathbb{Z}^{d}\right)$, see Theorem 14 in Sect. 3 . Then we apply this error estimate to two examples of general lattice rules, to so-called Kronecker lattices (Sect. 4.1) and to rank1 lattice point sets (Sect. 4.2). In both cases we achieve a convergence rate of order $O\left(N^{-s}\right)$ for the worst-case error up to $\log N$-terms. The major advantage of these constructions is that it is automatically clear which points belong to the unit cube. Hence, the proposed integration algorithms (which are in fact QMC rules) can be implemented very efficiently.

\subsection{Basic notation}

Throughout the paper $d \in \mathbb{N}$ denotes the dimension. By log we denote the dyadic logarithm. For $x \in \mathbb{R}$ we denote by $\{x\}$ the fractional part of $x$ and by $\langle x\rangle$ the distance of $x$ to the

1 It is known that $N \sim\left|\operatorname{det}\left(T^{-1}\right)\right|$ as the shrinking factor $a$ tends to infinity. This can be quantified as $|N-| \operatorname{det}\left(T^{-1}\right)|| \lesssim \log ^{d-1}\left(1+a^{d}\right)$ for all $a>1$, see Skriganov [8, Theorem 1.1]. 
nearest integer, i.e., $\langle x\rangle:=\min _{m \in \mathbb{Z}}|x-m|$. For $\boldsymbol{x}=\left(x_{1}, \ldots, x_{d}\right) \in \mathbb{R}^{d}$ and $p>0$ let $|\boldsymbol{x}|_{p}=\left(\sum_{i=1}^{d}\left|x_{i}\right|^{p}\right)^{1 / p}$ with the usual modification in the case $p=\infty$.

For $\ell=\left(\ell_{1}, \ldots, \ell_{d}\right) \in \mathbb{N}_{0}^{d}$ we denote by

$$
D^{\ell} f=\frac{\partial^{|\ell|}}{\partial x_{1}^{\ell_{1}} \partial x_{2}^{\ell_{2}} \cdots \partial x_{d}^{\ell_{d}}} f
$$

the operator of partial differentiation with $|\ell|=\ell_{1}+\cdots+\ell_{d}$. Furthermore, for $\xi \in \mathbb{R}^{d}$ let $e_{\xi}: \mathbb{R}^{d} \rightarrow \mathbb{C}, e_{\xi}(\boldsymbol{x}):=\exp (2 \pi i\langle\xi, \boldsymbol{x}\rangle)$, where $\langle\cdot, \cdot\rangle$ denotes the usual inner product in $\mathbb{R}^{d}$.

Before we formulate the main results of this work in more detail we need some preparation, which is presented in Sect. 2.

\section{Preliminaries}

\subsection{Basics of Fourier analysis}

Let $L_{p}=L_{p}\left(\mathbb{R}^{d}\right), 0<p \leq \infty$, be the space of all measurable functions $f: \mathbb{R}^{d} \rightarrow \mathbb{C}$ such that

$$
\|f\|_{p}:=\left(\int_{\mathbb{R}^{d}}|f(\boldsymbol{x})|^{p} \mathrm{~d} \boldsymbol{x}\right)^{1 / p}<\infty,
$$

with the usual modification if $p=\infty$. We will also need $L_{p}$-spaces on compact domains $\Omega \subset \mathbb{R}^{d}$ instead of the entire $\mathbb{R}^{d}$. We write $\|f\|_{L_{p}(\Omega)}$ for the corresponding (restricted) $L_{p}$-norm.

For $f \in L_{1}\left(\mathbb{R}^{d}\right)$ we define the Fourier transform

$$
\mathcal{F} f(\xi)=\int_{\mathbb{R}^{d}} f(\boldsymbol{y}) \overline{e_{\xi}(\boldsymbol{y})} \mathrm{d} \boldsymbol{y}, \quad \xi \in \mathbb{R}^{d},
$$

and the corresponding inverse Fourier transform $\mathcal{F}^{-1} f(\xi)=\mathcal{F} f(-\xi)$. Additionally, we define the spaces of continuous functions $C\left(\mathbb{R}^{d}\right)$, infinitely differentiable functions $C^{\infty}\left(\mathbb{R}^{d}\right)$ and infinitely differentiable functions with compact support $C_{0}^{\infty}\left(\mathbb{R}^{d}\right)$ as well as the $S c h$ wartz space $\mathcal{S}=\mathcal{S}\left(\mathbb{R}^{d}\right)$ of all rapidly decaying infinitely differentiable functions on $\mathbb{R}^{d}$, i.e.,

$$
\mathcal{S}:=\left\{\varphi \in C^{\infty}\left(\mathbb{R}^{d}\right):\|\varphi\|_{k, \ell}<\infty \text { for all } \boldsymbol{k}, \boldsymbol{\ell} \in \mathbb{N}_{0}^{d}\right\}
$$

where

$$
\|\varphi\|_{\boldsymbol{k}, \boldsymbol{\ell}}:=\left\|\prod_{j=1}^{d}\left(1+\left|x_{j}\right|\right)^{k_{j}}\left|D^{\ell} \varphi(\boldsymbol{x})\right|\right\|_{\infty} .
$$

The space $\mathcal{S}^{\prime}\left(\mathbb{R}^{d}\right)$, the topological dual of $\mathcal{S}\left(\mathbb{R}^{d}\right)$, is also referred to as the set of tempered distributions on $\mathbb{R}^{d}$. Indeed, a linear mapping $f: \mathcal{S}\left(\mathbb{R}^{d}\right) \rightarrow \mathbb{C}$ belongs to $\mathcal{S}^{\prime}\left(\mathbb{R}^{d}\right)$ if and only if there exist vectors $\boldsymbol{k}, \boldsymbol{\ell} \in \mathbb{N}_{0}^{d}$ and a constant $c=c_{f}$ such that

$$
|f(\varphi)| \leq c_{f}\|\varphi\|_{k, \ell}
$$

for all $\varphi \in \mathcal{S}\left(\mathbb{R}^{d}\right)$. The space $\mathcal{S}^{\prime}\left(\mathbb{R}^{d}\right)$ is equipped with the weak*-topology. The convolution $\varphi * \psi$ of two square-integrable functions $\varphi, \psi$ is defined via the integral

$$
(\varphi * \psi)(\boldsymbol{x})=\int_{\mathbb{R}^{d}} \varphi(\boldsymbol{x}-\boldsymbol{y}) \psi(\boldsymbol{y}) \mathrm{d} \boldsymbol{y} .
$$


If $\varphi, \psi \in \mathcal{S}\left(\mathbb{R}^{d}\right)$, then $\varphi * \psi$ still belongs to $\mathcal{S}\left(\mathbb{R}^{d}\right)$. In fact, the convolution operator can be extended to $\mathcal{S}\left(\mathbb{R}^{d}\right) \times L_{1}$, in which case we have $\varphi * \psi \in \mathcal{S}\left(\mathbb{R}^{d}\right)$, and to $\mathcal{S}\left(\mathbb{R}^{d}\right) \times \mathcal{S}^{\prime}\left(\mathbb{R}^{d}\right)$ via $(\varphi * f)(\boldsymbol{x})=f(\varphi(\boldsymbol{x}-\cdot))$. It makes sense point-wise and is a $C^{\infty}$-function in $\mathbb{R}^{d}$. As usual, the Fourier transform can be extended to $\mathcal{S}^{\prime}\left(\mathbb{R}^{d}\right)$ by $(\mathcal{F} f)(\varphi):=f(\mathcal{F} \varphi)$, where $f \in \mathcal{S}^{\prime}\left(\mathbb{R}^{d}\right)$ and $\varphi \in \mathcal{S}\left(\mathbb{R}^{d}\right)$. The mapping $\mathcal{F}: \mathcal{S}^{\prime}\left(\mathbb{R}^{d}\right) \rightarrow \mathcal{S}^{\prime}\left(\mathbb{R}^{d}\right)$ is a bijection.

\subsection{Function spaces}

In this section we introduce the function spaces under consideration, namely the Besov and Sobolev spaces of dominating mixed smoothness. There are several equivalent characterizations of these spaces, see [9]. For our purposes, the most suitable is the characterization by local means (see [9, Theorem 1.23] or [6, Section 4]).

We start with the definition of the spaces that are defined on $\mathbb{R}^{d}$. Let $\Psi_{0}, \Psi_{1} \in C_{0}^{\infty}(\mathbb{R})$ be such that

(i) $\left|\mathcal{F} \Psi_{0}(\xi)\right|>0$ for $|\xi|<\varepsilon$,

(ii) $\left|\mathcal{F} \Psi_{1}(\xi)\right|>0$ for $\frac{\varepsilon}{2}<|\xi|<2 \varepsilon$ and

(iii) $D^{\alpha} \mathcal{F} \Psi_{1}(0)=0 \quad$ for all $0 \leq \alpha \leq L$

for some $\varepsilon>0$. A suitable $L$ will be chosen in Definition 2. As usual, for $j \in \mathbb{N}$ we define

$$
\Psi_{j}(x)=2^{j-1} \Psi_{1}\left(2^{j-1} x\right),
$$

and the ( $d$-fold) tensorization

$$
\Psi_{\boldsymbol{m}}(\boldsymbol{x})=\prod_{i=1}^{d} \Psi_{m_{i}}\left(x_{i}\right),
$$

where $\boldsymbol{m}=\left(m_{1}, \ldots, m_{d}\right) \in \mathbb{N}_{0}^{d}$ and $\boldsymbol{x}=\left(x_{1}, \ldots, x_{d}\right) \in \mathbb{R}^{d}$.

Remark 1 There exist compactly supported functions $\Psi_{0}, \Psi_{1}$ satisfying (i)-(iii) above. Consider $\Psi_{0}$ to be the up-function, see Rvachev [10]. This function satisfies $\Psi_{0} \in C_{0}^{\infty}(\mathbb{R})$ with $\operatorname{supp}\left(\Psi_{0}\right)=[-1,1]$ and $\mathcal{F} \Psi_{0}(\xi)=\prod_{k=0}^{\infty} \operatorname{sinc}\left(2^{-k} \xi\right), \xi \in \mathbb{R}$, where sinc denotes the normalized sinus cardinalis $\operatorname{sinc}(\xi)=\sin (\pi \xi) /(\pi \xi)$. If we define $\Psi_{1} \in C_{0}^{\infty}(\mathbb{R})$ to be

$$
\Psi_{1}(x):=\frac{\mathrm{d}^{L}}{\mathrm{~d} x^{L}}\left(2 \Psi_{0}(2 \cdot)-\Psi_{0}\right)(x), \quad x \in \mathbb{R},
$$

it follows that $\mathcal{F} \Psi_{1}(\xi)=(2 \pi i \xi)^{L}\left(\mathcal{F} \Psi_{0}(\xi / 2)-\mathcal{F} \Psi_{0}(\xi)\right)$. It is easily checked that these functions satisfy the conditions (i)-(iii) above. In particular, (i) and (ii) are satisfied with $\varepsilon=$ 1. Moreover, we have for all $\boldsymbol{m} \in \mathbb{N}_{0}^{d}$ that the tensorized functions $\Psi_{\boldsymbol{m}}$ satisfy $\operatorname{supp}\left(\Psi_{\boldsymbol{m}}\right) \subseteq$ $[-1,1]^{d}$. We will work with this choice in the sequel.

Let us continue with the definition of the Besov spaces $\mathbf{B}_{p, \theta}^{s}=\mathbf{B}_{p, \theta}^{s}\left(\mathbb{R}^{d}\right)$ defined on the entire $\mathbb{R}^{d}$.

Definition 2 (Besov space) Let $0<p, \theta \leq \infty, s \in \mathbb{R}$, and $\left\{\Psi_{\boldsymbol{m}}\right\}_{\boldsymbol{m} \in \mathbb{N}_{0}^{d}}$ be as above with $L+1>s$. The Besov space of dominating mixed smoothness $\mathbf{B}_{p, \theta}^{s}=\mathbf{B}_{p, \theta}^{s}\left(\mathbb{R}^{d}\right)$ is the set of all $f \in \mathcal{S}^{\prime}\left(\mathbb{R}^{d}\right)$ such that

$$
\|f\|_{\mathbf{B}_{p, \theta}^{s}}:=\left(\sum_{\boldsymbol{m} \in \mathbb{N}_{0}^{d}} 2^{s|\boldsymbol{m}|_{1} \theta}\left\|\Psi_{\boldsymbol{m}} * f\right\|_{p}^{\theta}\right)^{1 / \theta}<\infty
$$


with the usual modification for $\theta=\infty$.

Remark 3 Other important function spaces are Sobolev spaces of dominating mixed smoothness, which are denoted by $\mathbf{H}_{p}^{s}=\mathbf{H}_{p}^{s}\left(\mathbb{R}^{d}\right)(1<s<\infty)$. In the case $s \in \mathbb{N}$ these spaces can be normed by

$$
\|f\|_{\mathbf{H}_{p}^{s}}:=\left(\sum_{\substack{\ell \in \mathbb{N}_{0}^{d} \\|\ell|_{\infty} \leq s}}\left\|D^{\ell} f\right\|_{p}^{p}\right)^{1 / p} .
$$

Note that $\mathbf{H}_{2}^{s}=\mathbf{B}_{2,2}^{s}$, i.e., the Sobolev(-Hilbert) spaces $\mathbf{H}_{2}^{s}$ appear as the special case $p=$ $\theta=2$ in the Besov space scale $\mathbf{B}_{p, \theta}^{s}$, see e.g., [11, Chapt. 2]. Moreover, the spaces $\mathbf{B}_{p, p}^{s}$ with $1 \leq p<\infty$ and $s \notin \mathbb{N}$ are called Sobolev-Slobodeckij spaces.

Remark 4 Different choices of $\Psi_{0}, \Psi_{1}$ in Definition 2 lead to equivalent (quasi-)norms. In fact, it is not even necessary that $\Psi_{0}$ and $\Psi_{1}$ have compact support. However, for the proof of our results, this specific choice is crucial.

In this article we are interested in classes of functions which are supported in the unit cube $[0,1]^{d}$, i.e., we consider the subclasses of $\mathbf{B}_{p, \theta}^{s}\left(\mathbb{R}^{d}\right)$ and $\mathbf{H}_{p}^{s}\left(\mathbb{R}^{d}\right)$

$$
\stackrel{\circ}{\mathbf{B}}_{p, \theta}^{s}:=\left\{f \in \mathbf{B}_{p, \theta}^{s}\left(\mathbb{R}^{d}\right): \operatorname{supp}(f) \subset[0,1]^{d}\right\}
$$

and

$$
\stackrel{\circ}{\mathbf{H}}_{p}^{s}:=\left\{f \in \mathbf{H}_{p}^{s}\left(\mathbb{R}^{d}\right): \operatorname{supp}(f) \subset[0,1]^{d}\right\} .
$$

The next lemma collects some frequently used embeddings between Besov and Sobolev spaces. They will be useful in obtaining our results for Sobolev spaces directly from the results for Besov spaces.

Lemma 5 Let $p \in(0, \infty]$, and let $s \in \mathbb{R}$.

(i) We have the chain of embeddings

$$
\mathbf{B}_{p, \min \{p, 2\}}^{s} \hookrightarrow \mathbf{H}_{p}^{s} \hookrightarrow \mathbf{B}_{p, \max \{p, 2\}}^{s} .
$$

(ii) For $p \geq 2$ we have the embedding

$$
\stackrel{\circ}{\mathbf{H}}_{p}^{s} \hookrightarrow \stackrel{\circ}{\mathbf{H}}_{2}^{s}=\stackrel{\circ}{\mathbf{B}}_{2,2}^{s} .
$$

Proof For a proof we refer to [11, Chapt. 2]. Note that for (ii) the compact support of the functions is necessary to ensure the corresponding embeddings of the $L_{p}$ spaces.

In the sequel we will always assume that $s>1 / p$. This ensures that the functions in $\mathbf{B}_{p, \theta}^{s}$ and $\mathbf{H}_{p}^{s}$, respectively, are continuous, see [11, Chapt. 2].

\subsection{Useful lemmas}

Here we collect some lemmas that will be essential for our analysis. For proofs and further literature for these results, we refer to [6, Sections 3.2 and 3.3].

The first lemma is a variant of Poisson's summation formula, see [12, Thm. VII.2.4]. Although this equality looks more technical than the original summation formula, this variant is exactly in the form we need and it comes with less assumptions on the involved functions. 
Lemma 6 (Poisson summation formula) Let $f \in L_{1}\left(\mathbb{R}^{d}\right)$ be continuous with compact support, $T \in \mathbb{R}^{d \times d}$ be an invertible matrix, and $B=\left(T^{-1}\right)^{\top}$. Furthermore, let $\varphi_{0} \in C_{0}^{\infty}(\mathbb{R})$ with $\varphi_{0}(0)=1$ and define $\varphi_{j}(t):=\varphi_{0}\left(2^{-j} t\right)-\varphi_{0}\left(2^{-j+1} t\right), j \in \mathbb{N}, t \in \mathbb{R}$, as well as the (tensorized) functions $\varphi_{\boldsymbol{m}}(\boldsymbol{x}):=\varphi_{m_{1}}\left(x_{1}\right) \cdots \varphi_{m_{d}}\left(x_{d}\right), \boldsymbol{m} \in \mathbb{N}_{0}^{d}, \boldsymbol{x} \in \mathbb{R}^{d}$. Then

$$
|\operatorname{det}(T)| \sum_{\ell \in \mathbb{Z}^{d}} f(T \boldsymbol{\ell})=\lim _{M \rightarrow \infty} \sum_{\boldsymbol{m}:|\boldsymbol{m}|_{\infty} \leq M} \sum_{\boldsymbol{k} \in \mathbb{Z}^{d}} \varphi_{\boldsymbol{m}}(B \boldsymbol{k}) \mathcal{F} f(B \boldsymbol{k}) .
$$

In particular, the limit on the right-hand side exists.

The lemma above holds for quite general choices of $\varphi_{0}$. However, we choose a certain $\varphi_{0}$ (and hence $\varphi_{m}$ ) that is related to the definition of our spaces, i.e., the functions $\Psi_{0}, \Psi_{1}$ from Sect. 2.2 are involved. This is because, given $\Psi_{0}$ and $\Psi_{1}$, we can construct a suitable decomposition of unity, i.e., a set of functions $\left\{\varphi_{j}\right\}_{j=0}^{\infty} \subset C_{0}^{\infty}(\mathbb{R})$ with

$$
\sum_{j=0}^{\infty} \varphi_{j}(x)=1 \text { for all } x \in \mathbb{R} .
$$

Lemma 7 Let $\Psi_{0}, \Psi_{1} \in \mathcal{S}(\mathbb{R})$ be functions with

$$
\left|\mathcal{F} \Psi_{0}(\xi)\right|>0 \text { for }|\xi|<\varepsilon
$$

and

$$
\left|\mathcal{F} \Psi_{1}(\xi)\right|>0 \quad \text { for } \frac{\varepsilon}{2}<|\xi|<2 \varepsilon
$$

for some $\varepsilon>0$. Then there exist $\Lambda_{0}, \Lambda_{1} \in \mathcal{S}(\mathbb{R})$ such that

(i) $\operatorname{supp} \mathcal{F} \Lambda_{0} \subset\{t \in \mathbb{R}:|t| \leq \varepsilon\}$,

(ii) $\operatorname{supp} \mathcal{F} \Lambda_{1} \subset\{t \in \mathbb{R}: \varepsilon / 2 \leq|t| \leq 2 \varepsilon\}$,

(iii) $\varphi_{0}:=\mathcal{F} \Lambda_{0} \cdot \mathcal{F} \Psi_{0} \in C_{0}^{\infty}(\mathbb{R})$ with $\varphi_{0}(0)=1$,

(iv) $\varphi_{j}(\cdot):=\varphi_{0}\left(2^{-j} \cdot\right)-\varphi_{0}\left(2^{-j+1} \cdot\right)=\mathcal{F} \Lambda_{j} \cdot \mathcal{F} \Psi_{j}, j \in \mathbb{N}$, where $\Psi_{j}(x)=$ $2^{j-1} \Psi_{1}\left(2^{j-1} x\right)$ and $\Lambda_{j}(x)=2^{j-1} \Lambda_{1}\left(2^{j-1} x\right)$.

Proof Following [9, Thm. 1.20], see also [6, Lemma 3.6], we define some function $\varphi \in$ $C_{0}^{\infty}(\mathbb{R})$ with $\varphi(t)=1$ if $|t| \leq 4 / 3$ and $\varphi(t)=0$ if $|t|>3 / 2$. Put $\Phi_{0}:=\mathcal{F}^{-1} \varphi$ and $\Phi_{1}:=2 \Phi_{0}(2 \cdot)-\Phi_{0}$, i.e., $\mathcal{F} \Phi_{1}=\mathcal{F} \Phi_{0}(\cdot / 2)-\mathcal{F} \Phi_{0}$. With $\Phi_{j}:=2^{j-1} \Phi_{1}\left(2^{j-1} \cdot\right)$ for $j \geq 1$ we define $\Lambda_{0}, \Lambda_{1}$ through

$$
\mathcal{F} \Lambda_{j}(t):=\frac{\mathcal{F} \Phi_{j}(2 t / \varepsilon)}{\mathcal{F} \Psi_{j}(t)} \quad \text { for } t \in \mathbb{R}
$$

Then (i) and (ii) follow from the support of $\mathcal{F} \Phi_{0}=\varphi$ and $\mathcal{F} \Phi_{1}$, (iii) comes from $\varphi_{0}(0)=$ $\mathcal{F} \Phi_{0}(0)=\varphi(0)=1$, and (iv) is easily shown by using the definition of $\Phi_{j}, j \geq 1$.

We define the $d$-fold tensorized functions

$$
\Lambda_{\boldsymbol{m}}(\boldsymbol{x}):=\prod_{i=1}^{d} \Lambda_{m_{i}}\left(x_{i}\right) \text { and } \Psi_{\boldsymbol{m}}(\boldsymbol{x}):=\prod_{i=1}^{d} \Psi_{m_{i}}\left(x_{i}\right) \quad \text { for } \boldsymbol{x} \in \mathbb{R}^{d} \text { and } \boldsymbol{m} \in \mathbb{N}_{0}^{d},
$$

where $\Lambda_{j}, \Psi_{j}, j \in \mathbb{N}_{0}$, are defined in Lemma 7. We obtain from Lemma 7 that we can use the functions

$$
\varphi_{\boldsymbol{m}}(\boldsymbol{x}):=\mathcal{F} \Lambda_{\boldsymbol{m}}(\boldsymbol{x}) \cdot \mathcal{F} \Psi_{\boldsymbol{m}}(\boldsymbol{x}) \quad \text { for } \boldsymbol{x} \in \mathbb{R}^{d},
$$


in Lemma 6. Moreover, by the construction of the tensorized functions and Lemma 7, we know that the support of $\mathcal{F} \Lambda_{\boldsymbol{m}}, \boldsymbol{m} \in \mathbb{N}_{0}^{d}$, is of the form

$$
I_{\boldsymbol{m}}:=\operatorname{supp} \mathcal{F} \Lambda_{\boldsymbol{m}} \subset\left\{\boldsymbol{x} \in \mathbb{R}^{d}: \frac{1}{2}\left\lfloor 2^{m_{i}-1}\right\rfloor \leq\left|x_{i}\right| \leq 2^{m_{i}}, i=1, \ldots, d\right\} .
$$

For this, recall that we choose $\Psi_{0}, \Psi_{1}$ such that $\varepsilon=1$, see Remark 1 .

As one can see in the lemmas above, we are concerned with sums of certain function evaluations of Fourier transforms. Finally, we want to bound such sums by the norm of the involved functions, i.e., we have to control the dependence on the matrix $B$, see Lemma 6 . For this we need the following two lemmas, see [6, Lemmas 3.3 and 3.5].

Lemma 8 Let $B \in \mathbb{R}^{d \times d}$ be an invertible matrix and $\Omega \subset \mathbb{R}^{d}$ be a bounded set. Furthermore, let $f \in \mathcal{S}\left(\mathbb{R}^{d}\right)$ with $\operatorname{supp}(f) \subset \Omega$ and define

$$
M_{B, \Omega}:=\left|\left\{\ell \in \mathbb{Z}^{d}:\left(\ell+[0,1)^{d}\right) \cap B^{\top}(\Omega) \neq \emptyset\right\}\right| .
$$

Then, for $1 \leq p \leq \infty$, we have

$$
\left\|\sum_{\ell \in \mathbb{Z}^{d}} \mathcal{F} f(B \ell) e_{\ell}\right\|_{L_{p}\left([0,1]^{d}\right)} \leq\left(\frac{M_{B, \Omega}}{|\operatorname{det}(B)|}\right)^{1-1 / p}\|f\|_{p} .
$$

Remark 9 Note that $M_{B, \Omega}$ is the number of unit cubes in the standard tessellation of $\mathbb{R}^{d}$ that are necessary to cover the set $B^{\top}(\Omega)$, while $|\operatorname{det}(B)|$ equals the volume of $B^{\top}\left([0,1]^{d}\right)$.

Lemma 10 Let $B \in \mathbb{R}^{d \times d}$ be an invertible matrix and $\Omega \in \mathbb{R}^{d}$ be a bounded set. Furthermore, let $g \in \mathcal{S}\left(\mathbb{R}^{d}\right)$ with $\operatorname{supp}(\mathcal{F} g) \subset \Omega$. Then, for $1 \leq p \leq \infty$, we have

$$
\left\|\sum_{\ell \in \mathbb{Z}^{d}} \mathcal{F} g(B \ell) e_{\ell}\right\|_{L_{p}\left([0,1]^{d}\right)} \leq\left|B\left(\mathbb{Z}^{d}\right) \cap \Omega\right|^{1-1 / p}\|g\|_{1} .
$$

\section{General error bound}

In this section, we provide a general upper bound on the error of general lattice rules for the integration in the spaces $\stackrel{\circ}{\mathbf{B}}_{p, \theta}^{s}$ of functions with support inside the unit cube. Here we mean by general lattice rules all algorithms of the form

$$
Q_{T}(f)=|\operatorname{det}(T)| \sum_{x \in T\left(\mathbb{Z}^{d}\right) \cap[0,1)^{d}} f(\boldsymbol{x})
$$

with an invertible matrix $T \in \mathbb{R}^{d \times d}$. Clearly, due to $\operatorname{supp}(f) \subset[0,1]^{d}$, we could also sum over all $\boldsymbol{x} \in T\left(\mathbb{Z}^{d}\right)$ without changing the value of $Q_{T}(f)$.

For an invertible matrix $T$ the dual lattice of the lattice $\mathbb{X}=T\left(\mathbb{Z}^{d}\right)$ is defined as $\mathbb{X}^{*}=$ $B\left(\mathbb{Z}^{d}\right):=T^{-\top}\left(\mathbb{Z}^{d}\right)$.

Remark 11 In the literature usually only those algorithms of the form (8) are called lattice rule that satisfy $T\left(\mathbb{Z}^{d}\right) \supset \mathbb{Z}^{d}$, see e.g., [13,14]. Such rules are a priori also suited for the integration of periodic functions, in contrast to the general algorithms of the form (8). We decided to use this nomenclature with the prefix "general" since it seems to be adequate for cubature rules that use function evaluations on a lattice. 
We prove the following theorem.

Theorem 12 Let $T \in \mathbb{R}^{d \times d}$ be invertible, $\mathbb{X}=T\left(\mathbb{Z}^{d}\right)$, $\mathbb{X}^{*}$ its dual lattice, $I_{\boldsymbol{m}}$ from (7) and $Q_{T}$ as in (8). Then, for $1 \leq p, \theta \leq \infty$ and $s>1 / p$, we have

$$
\operatorname{wce}\left(Q_{T}, \stackrel{\circ}{\mathbf{B}}_{p, \theta}^{s}\right) \lesssim\left(\sum_{\boldsymbol{m} \in \mathbb{N}_{0}^{d}} 2^{-s|\boldsymbol{m}|_{1} \theta^{\prime}}\left|\mathbb{X}^{*} \cap I_{\boldsymbol{m}} \backslash\{\boldsymbol{0}\}\right|^{\theta^{\prime} / p}\right)^{1 / \theta^{\prime}},
$$

with $\theta^{\prime}=\theta /(\theta-1)$. The hidden constant only depends on the quantity $M_{B,[-1,2]} /|\operatorname{det}(B)|$, cf. Remark 9.

Proof From Lemma 6 with $\varphi_{\boldsymbol{m}}$ from (6) we have

$$
Q_{T}(f)=\sum_{\boldsymbol{m} \in \mathbb{N}_{0}^{d}} \sum_{\boldsymbol{k} \in \mathbb{Z}^{d}} \mathcal{F}\left[\Lambda_{\boldsymbol{m}} * \Psi_{\boldsymbol{m}}\right](B \boldsymbol{k}) \mathcal{F} f(B \boldsymbol{k}),
$$

where $B=T^{-\top}$. Actually, the outer sum is defined as a certain limit; however, we will see that this sum converges absolutely, which justifies this notation.

Using that $\left\langle e_{\boldsymbol{k}}, e_{\ell}\right\rangle_{L_{2}\left([0,1]^{d}\right)}=1$, if $\boldsymbol{k}=\boldsymbol{\ell}$, and 0 otherwise, where $\langle\cdot, \cdot\rangle_{L_{2}\left([0,1]^{d}\right)}$ is the usual inner product in $L_{2}\left([0,1]^{d}\right)$, and $I(f)=\mathcal{F} f(\mathbf{0})$ we obtain

$$
\begin{aligned}
\left|I(f)-Q_{T}(f)\right| & =\left|\sum_{\boldsymbol{m} \in \mathbb{N}_{0}^{d}} \sum_{\boldsymbol{k} \neq \mathbf{0}} \mathcal{F} \Lambda_{\boldsymbol{m}}(B \boldsymbol{k}) \mathcal{F} \Psi_{\boldsymbol{m}}(B \boldsymbol{k}) \mathcal{F} f(B \boldsymbol{k})\right| \\
& =\left|\sum_{\boldsymbol{m} \in \mathbb{N}_{0}^{d}} \sum_{\boldsymbol{k} \neq \mathbf{0}} \sum_{\boldsymbol{\ell} \neq \mathbf{0}} \mathcal{F} \Lambda_{\boldsymbol{m}}(B \boldsymbol{k}) \mathcal{F}\left[\Psi_{\boldsymbol{m}} * f\right](B \boldsymbol{\ell})\left\langle e_{\boldsymbol{k}}, e_{\ell}\right\rangle_{L_{2}\left([0,1]^{d}\right)}\right| \\
& =\left|\sum_{\boldsymbol{m} \in \mathbb{N}_{0}^{d}}\left\langle\sum_{\boldsymbol{k} \neq \mathbf{0}} \mathcal{F} \Lambda_{\boldsymbol{m}}(B \boldsymbol{k}) e_{\boldsymbol{k}}, \sum_{\boldsymbol{\ell} \neq \mathbf{0}} \mathcal{F}\left[\Psi_{\boldsymbol{m}} * f\right](B \boldsymbol{\ell}) e_{\ell}\right\rangle_{L_{2}\left([0,1]^{d}\right)}\right| .
\end{aligned}
$$

By Hölder's inequality as well as Lemmas 8 and 10 we have

$$
\begin{aligned}
\left|I(f)-Q_{T}(f)\right| & \leq \sum_{\boldsymbol{m} \in \mathbb{N}_{0}^{d}}\left\|\sum_{\boldsymbol{k} \neq \mathbf{0}} \mathcal{F} \Lambda_{\boldsymbol{m}}(B \boldsymbol{k}) e_{\boldsymbol{k}}\right\|_{L_{p^{\prime}}\left([0,1]^{d}\right)}\left\|\sum_{\boldsymbol{\ell} \neq \mathbf{0}} \mathcal{F}\left[\Psi_{\boldsymbol{m}} * f\right](B \boldsymbol{\ell}) e_{\ell}\right\|_{L_{p}\left([0,1]^{d}\right)} \\
& \leq \sum_{\boldsymbol{m} \in \mathbb{N}_{0}^{d}}\left|\mathbb{X}^{*} \cap I_{\boldsymbol{m}} \backslash\{\mathbf{0}\}\right|^{1-1 / p^{\prime}}\left\|\Lambda_{\boldsymbol{m}}\right\|_{1}\left(\frac{M_{B, \operatorname{supp}\left(\Psi_{\boldsymbol{m}} * f\right)}}{|\operatorname{det}(B)|}\right)^{1-1 / p}\left\|\Psi_{\boldsymbol{m}} * f\right\|_{p}
\end{aligned}
$$

with $1 / p+1 / p^{\prime}=1$. For this note that, by construction, $\mathcal{F} \Lambda_{m}$ and $\Psi_{m} * f$ have compact support. Further $\operatorname{supp}\left(\Psi_{m} * f\right) \subset[-1,2]^{d}$ holds for all $\boldsymbol{m}$, see Remark 1 . Since, again by construction, $\left\|\Lambda_{m}\right\|_{1} \lesssim 1$, we finally obtain

$$
\left|I(f)-Q_{T}(f)\right| \lesssim\left(\sum_{\boldsymbol{m} \in \mathbb{N}_{0}^{d}} 2^{-s|\boldsymbol{m}|_{1} \theta^{\prime}}\left|\mathbb{X}^{*} \cap I_{\boldsymbol{m}} \backslash\{\boldsymbol{0}\}\right|^{\theta^{\prime} / p}\right)^{1 / \theta^{\prime}}\|f\|_{\dot{\mathbf{B}}_{p, \theta}^{s}}
$$

with $1 / \theta+1 / \theta^{\prime}=1$, see Definition 2 .

This shows that, in order to prove bounds on the worst-case error, it is enough to study the numbers $\left|\mathbb{X}^{*} \cap I_{\boldsymbol{m}} \backslash\{\boldsymbol{0}\}\right|$ for $\boldsymbol{m} \in \mathbb{N}_{0}^{d}$. Moreover, as the next lemma shows, this quantity 
can be bounded by the Zaremba index of the lattice $\mathbb{X}$, which is, loosely speaking, the largest $\ell \in \mathbb{N}$ such that $\left|\mathbb{X}^{*} \cap I_{\boldsymbol{m}} \backslash\{\boldsymbol{0}\}\right|=0$ for all $\boldsymbol{m}$ with $|\boldsymbol{m}|_{1} \leq \ell$. More precisely, we define the Zaremba index of the lattice $\mathbb{X}$ by

$$
\rho(\mathbb{X}):=\inf _{z \in \mathbb{X}^{*} \backslash\{\mathbf{0}\}} r(z),
$$

where, for $z=\left(z_{1}, \ldots, z_{d}\right), r(z):=\prod_{j=1}^{d} \bar{z}_{j}$ with $\bar{z}:=\max \{1,|z|\}$. The connection of the Zaremba index to numerical integration is well known, see e.g., [13-17] and the references therein. However, these classical results are for Korobov spaces or star discrepancy. In this paper we are concerned with Besov spaces of dominating mixed smoothness. We repeat the (relatively short) proofs here for convenience of the reader.

Lemma 13 Let $T \in \mathbb{R}^{d \times d}$ be invertible, $\mathbb{X}=T\left(\mathbb{Z}^{d}\right)$, $\mathbb{X}^{*}$ its dual lattice and $I_{\boldsymbol{m}}$ from (7). Then, for all $\boldsymbol{m} \in \mathbb{N}_{0}^{d}$, we have

$$
\left|\mathbb{X}^{*} \cap I_{\boldsymbol{m}} \backslash\{\mathbf{0}\}\right| \lesssim \begin{cases}0 & \text { if }|\boldsymbol{m}|_{1}<\log (\rho(\mathbb{X})), \\ 2^{|\boldsymbol{m}|_{1}} / \rho(\mathbb{X}) & \text { otherwise, }\end{cases}
$$

where log denotes the dyadic logarithm.

Proof Let $M:=\log (\rho(\mathbb{X}))$. For $\boldsymbol{x} \in I_{\boldsymbol{m}}$, we have $r(\boldsymbol{x}) \leq 2^{|\boldsymbol{m}|_{1}}$. This shows that, for $|\boldsymbol{m}|_{1}<M$, there is no $\boldsymbol{x} \in \mathbb{X}^{*}$ in $I_{\boldsymbol{m}}$, except possibly the origin. This proves the first bound.

By the same argument it is clear, that also boxes of the form $\left\{x \in \mathbb{R}^{d}:\left|x_{i}\right| \leq 2^{\ell_{i}}, i=\right.$ $1, \ldots, d\}$ with $|\ell|_{1}<M$ do not contain points from $\mathbb{X}^{*} \backslash\{\mathbf{0}\}$. If we halve all sides of this set, i.e., if we consider the sets

$$
J_{\ell}:=\left\{\boldsymbol{x} \in \mathbb{R}^{d}: 0 \leq x_{i} \leq 2^{\ell_{i}}, i=1, \ldots, d\right\},
$$

it is easy to see that all translates of $J_{\ell},|\ell|_{1}<M$, contain at most one $\boldsymbol{x} \in \mathbb{X}^{*}$.

Now consider $|\boldsymbol{m}|_{1} \geq M$. Then there exists an $\ell \in \mathbb{N}_{0}^{d}$ with $|\ell|_{1}=\lceil M\rceil-1<M$ such that $\boldsymbol{m}-\boldsymbol{\ell} \in \mathbb{N}_{0}^{d}$. Clearly, $I_{\boldsymbol{m}} \subset\left\{\boldsymbol{x} \in \mathbb{R}^{d}:\left|x_{i}\right| \leq 2^{m_{i}}, i=1, \ldots, d\right\}$ can be covered by $2^{|\boldsymbol{m}-\ell|_{1}+d}=2^{|\boldsymbol{m}|_{1}-\lceil M\rceil+d+1} \leq 2^{|\boldsymbol{m}|_{1}+d+1} / \rho(\mathbb{X})$ translates of $J_{\ell}$, each containing at most one $\boldsymbol{x} \in \mathbb{X}^{*}$. This proves the second bound.

Together with Theorem 12 this implies the following.

Theorem 14 Let $T \in \mathbb{R}^{d \times d}$ be invertible with $d_{T}:=1 /|\operatorname{det}(T)| \geq 1, \mathbb{X}=T\left(\mathbb{Z}^{d}\right)$ and $Q_{T}$ as in (8). Then, for $1 \leq p, \theta \leq \infty$ and $s>1 / p$, we have

$$
\operatorname{wce}\left(Q_{T}, \stackrel{\circ}{\mathbf{B}}_{p, \theta}^{s}\right) \lesssim \rho(\mathbb{X})^{-s}\left(1+\log \left(d_{T}\right)\right)^{(d-1)(1-1 / \theta)} .
$$

The hidden constant only depends on the quantity $M_{B,[-1,2]^{d}} /|\operatorname{det}(B)|$, see Remark 9.

Proof Let $M:=\log (\rho(\mathbb{X}))$ and note that $\left|\left\{\boldsymbol{m} \in \mathbb{N}_{0}^{d}:|\boldsymbol{m}|_{1}=\ell\right\}\right| \leq(\ell+1)^{d-1}$. By Theorem 12 and Lemma 13 we have

$$
\begin{aligned}
\operatorname{wce}\left(Q_{T}, \stackrel{\circ}{\mathbf{B}}_{p, \theta}^{s}\right) & \lesssim\left(\sum_{\boldsymbol{m} \in \mathbb{N}_{0}^{d}} 2^{-s|\boldsymbol{m}|_{1} \theta^{\prime}}\left|\mathbb{X}^{*} \cap I_{\boldsymbol{m}} \backslash\{\boldsymbol{0}\}\right|^{\theta^{\prime} / p}\right)^{1 / \theta^{\prime}} \\
& \lesssim \rho(\mathbb{X})^{-1 / p}\left(\sum_{\boldsymbol{m}:|\boldsymbol{m}|_{1} \geq M} 2^{(1 / p-s)|\boldsymbol{m}|_{1} \theta^{\prime}}\right)^{1 / \theta^{\prime}}
\end{aligned}
$$




$$
\begin{aligned}
& \leq \rho(\mathbb{X})^{-1 / p}\left(\sum_{\ell=\lceil M\rceil}^{\infty}(\ell+1)^{d-1} 2^{(1 / p-s) \ell \theta^{\prime}}\right)^{1 / \theta^{\prime}} \\
& \lesssim \rho(\mathbb{X})^{-1 / p} 2^{M(1 / p-s)}(M+1)^{(d-1) / \theta^{\prime}}\left(\sum_{\ell=1}^{\infty}(\ell+1)^{d-1} 2^{(1 / p-s) \ell \theta^{\prime}}\right)^{1 / \theta^{\prime}}
\end{aligned}
$$

It follows from Minkowski's theorem that $\rho(\mathbb{X}) \leq\left|\operatorname{det}\left(T^{-1}\right)\right|$, and hence $M \leq \log \left(d_{T}\right)$. This proves the result since $s>1 / p$.

\section{Application to specific point sets}

In this section we apply the general results from the last section to specific point sets. More precisely, we study Kronecker lattices and rank-1 lattice point sets in dimensions $d \geq 2$. By Theorem 14 it is enough to bound the Zaremba index of these lattices. However, since the bounds on the Zaremba index are worse in higher dimensions, we treat the lower-dimensional cases separately.

\subsection{Kronecker lattices}

We study Kronecker lattices which are point sets of the form

$$
\mathcal{P}_{N}(\boldsymbol{\alpha})=\left\{\left(\frac{n}{N},\left\{n \alpha_{1}\right\}, \ldots,\left\{n \alpha_{d-1}\right\}\right): n=0,1, \ldots, N-1\right\},
$$

where $\boldsymbol{\alpha}=\left(\alpha_{1}, \ldots, \alpha_{d-1}\right) \in \mathbb{R}^{d-1}$. These point sets can be written as

$$
\mathcal{P}_{N}(\boldsymbol{\alpha})=\mathbb{X}_{N}(\boldsymbol{\alpha}) \cap[0,1)^{d},
$$

where $\mathbb{X}_{N}(\boldsymbol{\alpha})=T_{N}\left(\mathbb{Z}^{d}\right)$ and

$$
T_{N}=T_{N}(\boldsymbol{\alpha})=\left(\begin{array}{ccccc}
0 & \cdots & \cdots & 0 & 1 / N \\
1 & 0 & \cdots & 0 & \alpha_{1} \\
0 & \ddots & \ddots & \vdots & \vdots \\
\vdots & \ddots & \ddots & 0 & \vdots \\
0 & \cdots & 0 & 1 & \alpha_{d-1}
\end{array}\right) \in \mathbb{R}^{d \times d}
$$

Note that $\operatorname{det}\left(T_{N}\right)=(-1)^{d+1} / N$ and hence $d_{T_{N}}=N$. Hence, we can use the results from the last section to prove upper bounds on the error of the cubature rule $Q_{T_{N}}$. Recall that the dual lattice of $\mathbb{X}_{N}(\boldsymbol{\alpha})$ is $\mathbb{X}_{N}^{*}(\boldsymbol{\alpha}):=B_{N}\left(\mathbb{Z}^{d}\right)$ with

$$
B_{N}=B_{N}(\boldsymbol{\alpha})=\left(T_{N}^{\top}\right)^{-1}=\left(\begin{array}{ccccc}
-N \alpha_{1} & -N \alpha_{2} & \ldots & -N \alpha_{d-1} & N \\
1 & 0 & \ldots & 0 & 0 \\
0 & \ddots & \ddots & \vdots & \vdots \\
\vdots & \ddots & \ddots & 0 & 0 \\
0 & \ldots & 0 & 1 & 0
\end{array}\right) \text {. }
$$


Fig. 1 Covering of

$B_{N}^{\uparrow}\left([-1,2]^{2}\right)$ with unit-squares

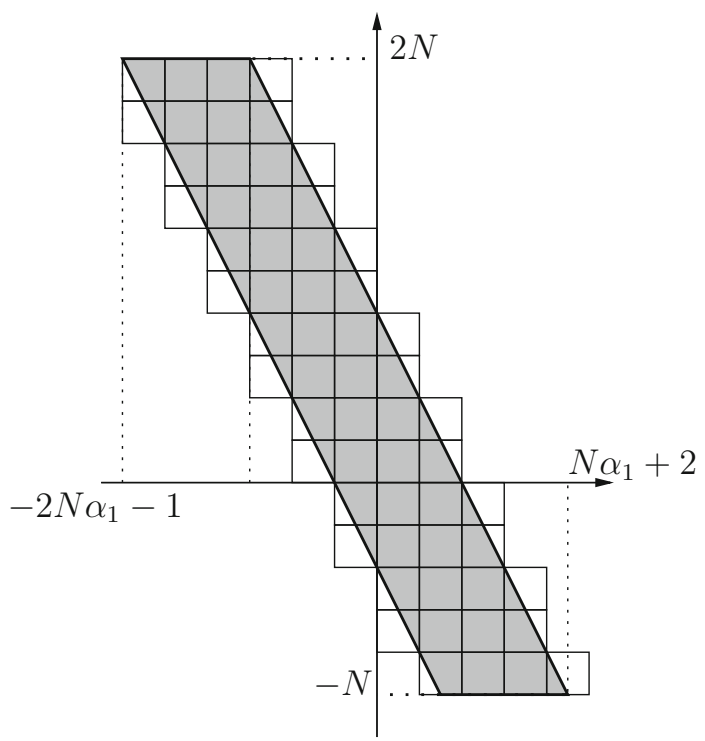

Remark 15 In view of Remark 9 the sequence of matrices $B_{N}, N \geq 1$, from (12) satisfies

$$
\sup _{N \geq 1} \frac{M_{B_{N},[-1,2]^{d}}}{\left|\operatorname{det}\left(B_{N}\right)\right|} \leq c_{\boldsymbol{\alpha}}<\infty .
$$

Thus we can apply Theorem 14 for the Kronecker lattices, ignoring the hidden constants. To see this note that $\left|\operatorname{det}\left(B_{N}\right)\right|=N$ and that $B_{N}^{\top}\left([-1,2]^{d}\right)$ is a $d$-dimensional oblique prism with translated copies of $[-1,2]^{d-1} \times\{0\}$ as base faces. The translation vector is $\left(N \alpha_{1}, \ldots, N \alpha_{d-1},-N\right)$ for the "bottom" base and $\left(-2 N \alpha_{1}, \ldots,-2 N \alpha_{d-1}, 2 N\right)$ for the "top" base and hence the height is $3 N$ (see Figure 1 for $d=2$ ).

Since all lattices that follow will be of this form we use the notation

$$
\rho_{d}(N, \boldsymbol{\alpha}):=\rho\left(\mathbb{X}_{N}(\boldsymbol{\alpha})\right) .
$$

Moreover, we write

$$
\operatorname{wce}\left(\mathcal{P}_{N}(\boldsymbol{\alpha}), \stackrel{\circ}{\mathbf{B}}_{p, \theta}^{s}\right):=\operatorname{wce}\left(Q_{T_{N}}, \stackrel{\circ}{\mathbf{B}}_{p, \theta}^{s}\right) .
$$

In the following we fix a vector $\boldsymbol{\alpha} \in \mathbb{R}^{d-1}$ and just let $N$ grow in (10). Given a "good" $\boldsymbol{\alpha}$, this makes the point set $\mathcal{P}_{N}(\boldsymbol{\alpha})$ particularly easy to implement, since one needs only $N d$ arithmetic operations.

It is not surprising, and well known, that bounds on the Zaremba index of lattices $\mathbb{X}_{N}(\boldsymbol{\alpha})$ depend on Diophantine approximation properties of the vector $\boldsymbol{\alpha}$. More precisely, a lattice has large Zaremba index if the involved numbers $\alpha_{1}, \ldots, \alpha_{d-1}$ are badly approximable (in a certain sense). This is reflected by the following lemma.

Lemma 16 Let $\boldsymbol{\alpha} \in \mathbb{R}^{d-1}$ and $\psi: \mathbb{N} \rightarrow \mathbb{R}^{+}$be non-decreasing such that

$$
\left(\prod_{j=1}^{d-1} \bar{k}_{j} \psi\left(\bar{k}_{j}\right)\right)\langle\boldsymbol{\alpha} \cdot \boldsymbol{k}\rangle \geq c=c(d, \boldsymbol{\alpha})>0
$$


for all $\boldsymbol{k}=\left(k_{1}, \ldots, k_{d-1}\right) \in \mathbb{Z}^{d-1} \backslash\{\mathbf{0}\}$, where $\langle x\rangle:=\min _{m \in \mathbb{Z}}|x-m|$ is the distance of $x \in \mathbb{R}$ to the nearest integer. Then, with $c^{\prime}=\min \left\{c, \psi(1)^{d-1}\right\}$, we have

$$
\rho_{d}(N, \boldsymbol{\alpha}) \geq \frac{c^{\prime} N}{\psi(N)^{d-1}} \quad \text { for all } N \geq 1
$$

Proof To bound $\rho_{d}(N, \boldsymbol{\alpha})$, we have to bound $r(\boldsymbol{z})$ uniformly over all $z \in \mathbb{X}_{N}^{*}(\boldsymbol{\alpha}) \backslash\{\boldsymbol{0}\}$, see (9). By definition of $\mathbb{X}_{N}^{*}(\boldsymbol{\alpha})$ and $B_{N}$, we have for $z:=B_{N} \boldsymbol{k}^{\prime} \in \mathbb{X}_{N}^{*}(\boldsymbol{\alpha}) \backslash\{\boldsymbol{0}\}, \boldsymbol{k}^{\prime}=\left(k_{1}, \ldots, k_{d}\right) \in$ $\mathbb{Z}^{d}$, that

$$
r(z)=\prod_{j=1}^{d} \overline{\left(B_{N} \boldsymbol{k}^{\prime}\right)_{j}}=\left(\prod_{j=1}^{d-1} \bar{k}_{j}\right) \overline{\left(-N \alpha_{1} k_{1}-\cdots-N \alpha_{d-1} k_{d-1}+N k_{d}\right)} .
$$

Since $\boldsymbol{z} \neq \mathbf{0}$ we have $\boldsymbol{k}^{\prime} \neq \mathbf{0}$. We distinguish three cases.

First assume that $k_{1}=\cdots=k_{d-1}=0$ and hence $k_{d} \neq 0$. This already implies $r(z) \geq N$.

Next assume that $\left|k_{j}\right| \geq N$ for some $j=1, \ldots, d-1$. Then it follows from the definition that $r(z) \geq \bar{k}_{j} \geq N$.

Finally, assume that $\boldsymbol{k}:=\left(k_{1}, \ldots, k_{d-1}\right) \in \mathbb{Z}^{d-1} \backslash\{\mathbf{0}\}$ with $|\boldsymbol{k}|_{\infty} \leq N$. Clearly, by choosing the right $k_{d} \in \mathbb{Z}$, we have by assumption

$$
\begin{aligned}
r(z) & \geq N\left(\prod_{j=1}^{d-1} \bar{k}_{j}\right)\left|-\alpha_{1} k_{1}-\cdots-\alpha_{d-1} k_{d-1}+k_{d}\right| \geq N\left(\prod_{j=1}^{d-1} \bar{k}_{j}\right)\langle\boldsymbol{\alpha} \cdot \boldsymbol{k}\rangle \\
& \geq \frac{c N}{\prod_{j=1}^{d-1} \psi\left(\bar{k}_{j}\right)} \geq \frac{c N}{\psi(N)^{d-1}} .
\end{aligned}
$$

This shows that in any case $r(z) \geq \min \left\{N, c N / \psi(N)^{d-1}\right\} \geq N / \psi(N)^{d-1} \min \left\{\psi(1)^{d-1}, c\right\}$ and thus proves the claim.

We now treat the cases $d=2$ and $d \geq 3$ separately, since the known results on the existence of (simultaneously) badly approximable numbers differ in these cases.

\subsubsection{The case $d=2$}

We say that a real number $\alpha$ is badly approximable, if there is a positive constant $c_{0}=$ $c_{0}(\alpha)>0$ such that

$$
k\langle k \alpha\rangle \geq c_{0}>0 \quad \text { for all integers } k \geq 1 .
$$

It is well known that an irrational number $\alpha$ is badly approximable if and only if the sequence $a_{1}, a_{2}, a_{3}, \ldots$ of partial quotients in the continued fraction expansion of $\alpha=$ $\left[a_{0} ; a_{1}, a_{2}, a_{3}, \ldots\right]$ is bounded, i.e., there is some $M=M(\alpha)>0$ such that $a_{j} \leq M$ for all integers $j \geq 1$, see e.g., [18]. For example for the golden ratio $\alpha=(1+\sqrt{5}) / 2$ we have that the continued fraction coefficients are all 1 and hence of course are also bounded.

It is easily seen from the definition of badly approximable numbers that we can apply Lemma 16 with $d=2$ and $\psi \equiv 1$, which proves that, for the constant $c_{0}^{\prime}=\min \left(1, c_{0}\right)$,

$$
\rho_{2}(N, \alpha) \geq c_{0}^{\prime} N
$$

for all $N \in \mathbb{N}$. This implies the following theorem. 
Theorem 17 Let $\alpha$ be a badly approximable number, $N \in \mathbb{N}$ and $\mathcal{P}_{N}(\alpha)$ as in (10). Then, for $1 \leq p, \theta \leq \infty$ and $s>1 / p$,

$$
\operatorname{wce}\left(\mathcal{P}_{N}(\alpha), \stackrel{\circ}{\mathbf{B}}_{p, \theta}^{s}\right) \asymp \frac{(\log N)^{1-1 / \theta}}{N^{s}} .
$$

Proof The upper bound follows from Theorem 14 and the lower bound was proven, e.g., in [6, Theorem 7.3].

\subsubsection{The case $d \geq 3$}

Unfortunately, in dimensions greater than two the results are not as satisfactory as for $d=2$. That is, we do not know if vectors $\alpha$ exist that give the optimal order of convergence of the corresponding (general) lattice rule.

Assume for the moment that we have a vector $\alpha \in \mathbb{R}^{d-1}$ for $d \geq 3$ such that

$$
r(\boldsymbol{k})\langle\boldsymbol{\alpha} \cdot \boldsymbol{k}\rangle>c=c(\boldsymbol{\alpha})>0 \quad \text { for all } \boldsymbol{k} \in \mathbb{Z}^{d-1} \backslash\{\boldsymbol{0}\} .
$$

In this case we could show that $\operatorname{wce}\left(\mathcal{P}_{N}(\boldsymbol{\alpha}), \stackrel{\circ}{\mathbf{B}}_{p, \theta}^{s}\right) \lesssim(\log N)^{(d-1)(1-1 / \theta)} / N^{s}$, which is the optimal order of convergence. However, a famous conjecture of Littlewood states that there is no $\alpha \in \mathbb{R}^{d-1}, d \geq 3$, with this property, see e.g., [19]. See also [20] for a discussion of this Diophantine problem in the context of the discrepancy of $(n \boldsymbol{\alpha})$-sequences.

The best we can hope for at the moment for our problem are metrical results. These are based on the following lemma.

Lemma 18 Let $\psi: \mathbb{N} \rightarrow \mathbb{R}^{+}$be non-decreasing such that the series $\sum_{n \geq 1} \frac{1}{n \psi(n)}<\infty$. Then for almost every $\alpha \in \mathbb{R}^{d-1}$ and every $N \geq 1$ we have

$$
\rho_{d}(N, \boldsymbol{\alpha}) \geq \frac{c N}{\psi(N)^{d-1}}
$$

for some $c>0$. For example, we can choose $\psi(N)=(\log N)(\log \log N)^{1+\delta}$ for arbitrary $\delta>0$ for $N \geq 3$ and $\psi(N)=1$ for $N<3$.

Proof From [21, Lemma 5] we obtain that, under the assumptions of the lemma, we can apply Lemma 16 for almost every $\alpha \in \mathbb{R}^{d-1}$.

This implies the following result.

Theorem 19 Let $\psi: \mathbb{N} \rightarrow \mathbb{R}^{+}$be non-decreasing such that $\sum_{n \geq 1} \frac{1}{n \psi(n)}<\infty$. Then, for almost all $\boldsymbol{\alpha} \in \mathbb{R}^{d-1}$ and every $N \geq 1$ we have

$$
\operatorname{wce}\left(\mathcal{P}_{N}(\boldsymbol{\alpha}), \stackrel{\circ}{\mathbf{B}}_{p, \theta}^{s}\right) \lesssim \frac{(\log N)^{(d-1)(1-1 / \theta)}}{N^{s}} \psi(N)^{s(d-1)} .
$$

For example, for $\delta>0$ for almost all $\alpha \in \mathbb{R}^{d-1}$ we have

$$
\operatorname{wce}\left(\mathcal{P}_{N}(\boldsymbol{\alpha}), \stackrel{\circ}{\mathbf{B}}_{p, \theta}^{s}\right) \lesssim \frac{(\log N)^{(d-1)(s+1-1 / \theta)}}{N^{s}}(\log \log N)^{s(d-1)(1+\delta)} .
$$

Remark 20 In dimension $d=3$ the metrical result can be slightly improved. It follows from results in [19] that there exist $\left(\alpha_{1}, \alpha_{2}\right) \in \mathbb{R}^{2}$ such that the assumption of Lemma 16 holds with $\psi(N)=(\log N) \log \log N$. Hence, for $d=3$, the second statement of Theorem 19 holds with $\delta=0$. 
However, if we want a result for concrete $\alpha \in \mathbb{R}^{d-1}$ for $d \geq 3$, the situation is even worse. Recall that for a real number $\eta$, a $(d-1)$-tuple $\alpha \in(\mathbb{R} \backslash \mathbb{Q})^{d-1}$ is said to be of approximation type $\eta$, if $\eta$ is the infimum of all numbers $\sigma$ for which there exists a positive constant $c=c(\sigma, \boldsymbol{\alpha})$ such that

$$
r(\boldsymbol{h})^{\sigma}\langle\boldsymbol{h} \cdot \boldsymbol{\alpha}\rangle \geq c \quad \text { for all } \boldsymbol{h} \in \mathbb{Z}^{d-1} \backslash\{\boldsymbol{0}\} .
$$

It is well known that the type $\eta$ of an irrational vector $\boldsymbol{\alpha}$ is at least one. On the other hand it has been shown by Schmidt [22] that $\alpha=\left(\alpha_{1}, \ldots, \alpha_{d-1}\right)$, with real algebraic components for which $1, \alpha_{1}, \ldots, \alpha_{d-1}$ are linearly independent over $\mathbb{Q}$, is of type $\eta=1$. In particular, $\left(\mathrm{e}^{r_{1}}, \ldots, \mathrm{e}^{r_{d-1}}\right)$ with distinct nonzero rationals $r_{1}, \ldots, r_{d-1}$ or $\left(\sqrt{p_{1}}, \ldots, \sqrt{p_{d-1}}\right)$ with distinct prime numbers $p_{1}, \ldots, p_{d-1}$ are of type $\eta=1$.

From (14), Lemma 16 and Theorem 14, we obtain the following result.

Theorem 21 Let $\alpha \in(\mathbb{R} \backslash \mathbb{Q})^{d-1}$ be of approximation type 1. Then for every $\delta>0$ we have

$$
\operatorname{wce}\left(\mathcal{P}_{N}(\boldsymbol{\alpha}), \stackrel{\circ}{\mathbf{B}}_{p, \theta}^{s}\right) \lesssim \frac{1}{N^{s-\delta}} .
$$

\subsection{Rank-1 lattice point sets}

A rank-1 lattice point set is given by the points $\left\{\frac{n}{N} g\right\}$ for $n=0,1, \ldots, N-1$, where $\boldsymbol{g}=\left(g_{0}, g_{1}, \ldots, g_{d-1}\right)$ is a lattice point in $\mathbb{Z}^{d}$ and where the fractional part is applied componentwise. We restrict ourselves to the case where $g_{0}=1$ (if $N$ is a prime number, this still covers all possible rank-1 lattice point sets). Then we can write a rank-1 lattice point set as

$$
\begin{aligned}
\mathcal{P}_{N}(\boldsymbol{g}) & =\mathbb{X}_{N}(\boldsymbol{g}) \cap[0,1)^{d} \\
& =\left\{\left(\frac{n}{N},\left\{n \frac{g_{1}}{N}\right\}, \ldots,\left\{n \frac{g_{d-1}}{N}\right\}\right): n=0,1, \ldots, N-1\right\},
\end{aligned}
$$

where $\mathbb{X}_{N}(\boldsymbol{g})=T_{N}\left(\mathbb{Z}^{d}\right)$ with

$$
T_{N}=T_{N}(\boldsymbol{g})=\left(\begin{array}{ccccc}
0 & \ldots & \ldots & 0 & 1 / N \\
1 & 0 & \ldots & 0 & g_{1} / N \\
0 & \ddots & \ddots & \vdots & \vdots \\
\vdots & \ddots & \ddots & 0 & \vdots \\
0 & \ldots & 0 & 1 & g_{d-1} / N
\end{array}\right) \in \mathbb{R}^{d \times d}
$$

In view of Sect. 4.1 we see that we replace the possibly irrational point $\alpha$ by the rational point $\boldsymbol{g} / N$. So, for consistent notation, we should have used, e.g., the denotation $\mathcal{P}_{N}(\boldsymbol{g} / N)$ for the point set. However, we use $\mathcal{P}_{N}(\boldsymbol{g})$ etc. for simplicity. For the same reasoning we let $\rho_{d}(N, \boldsymbol{g}):=\rho\left(\mathbb{X}_{N}(\boldsymbol{g} / N)\right)$. The Zaremba index of rank-1 lattice point sets is a well studied quantity, and we can use the known results in order to apply them in Theorem 14. Since the statement of Remark 15 holds also in this case, we can ignore the hidden constants in Theorem 14. Again we treat the cases $d=2$ and $d \geq 3$ separately.

Remark 22 For $d=2$ the construction is again based on the boundedness of the partial quotients of $g_{1} / N$. So, given a badly approximable number $\alpha$, see Sect. 4.1.1, one can use its convergents $p_{k} / q_{k}, k=1,2, \ldots$, to construct the (optimal) sequence of lattices $\mathcal{P}_{q_{k}}\left(\left(1, p_{k}\right)\right)$ with respect to the worst-case error. To find an analogous construction in higher dimensions is a challenging open problem. 


\subsubsection{The case $d=2$}

Let $g \in\{1, \ldots, N-1\}$ with $\operatorname{gcd}(g, N)=1$. Let $a_{1}, a_{2}, \ldots, a_{l}$ be the partial quotients in the continued fraction expansion of $g / N$ and let $K\left(\frac{g}{N}\right)=\max _{1 \leq j \leq l} a_{j}$. Then it was shown by Zaremba [23] (see also [13, Theorem 5.17]) that the Zaremba index $\rho_{2}(N, \boldsymbol{g})$ for $\boldsymbol{g}=(1, g)$ can be bounded in terms of $K(\mathrm{~g} / \mathrm{N})$, more precisely, that

$$
\frac{N}{K(g / N)+2} \leq \rho_{2}(N, \boldsymbol{g}) \leq \frac{N}{K(g / N)} .
$$

From this result in conjunction with Theorem 14 we obtain the following result:

Theorem 23 Let $N \in \mathbb{N}$ and $\boldsymbol{g}=(1, g)$ with $g \in\{1,2, \ldots, N-1\} \operatorname{such}$ that $\operatorname{gcd}(g, N)=1$ and $K(g / N) \leq C$ for some constant $C>0$. Then, for $1 \leq p, \theta \leq \infty$ and $s>1 / p$,

$$
\operatorname{wce}\left(\mathcal{P}_{N}(g), \stackrel{\circ}{\mathbf{B}}_{p, \theta}^{s}\right) \asymp \frac{(\log N)^{1-1 / \theta}}{N^{s}} .
$$

Remark 24 In particular the result holds for Fibonacci rules, where $N=F_{n}$ and $g=$ $F_{n-1}$, the $n$th and $(n-1)$ st Fibonacci numbers, respectively. In this case the continued fraction coefficients of $g / N=F_{n-1} / F_{n}$ are all exactly 1 . Fibonacci rules were also used by Temlyakov, see [24] and [3, Section 4.1].

Remark 25 Note that a famous conjecture of Zaremba [25, p. 76] states that for every integer $N \geq 2$ one can find a $g \in\{1, \ldots, N\}$ with $\operatorname{gcd}(g, N)=1$ such that the continued fraction coefficients of $g / N$ are bounded by some constant $K$ (in fact, he conjectured that $K=5$ ). Niederreiter [26] established this conjecture for all $N$ of the form $2^{m}, 3^{m}$ or $5^{m}$ for $m \in \mathbb{N}$. Bourgain and Kontorovich [27] proved Zaremba's conjecture for almost all $N$ with a constant $K=50$. Huang [28] improved this result to show Zaremba's conjecture for almost all $N$ with constant $K=5$.

\subsubsection{The case $d \geq 3$}

It follows from a result of Zaremba [29] that for every $d \geq 2$ and $N \geq 2$ there exists a lattice point $g \in \mathbb{Z}^{d}$ such that

$$
\rho_{d}(N, \boldsymbol{g}) \geq \frac{C_{d} N}{(\log N)^{d-1}}
$$

In fact, one can choose $C_{d}=(d-1) ! / 2^{d-1}$ (see also [13, Theorem 5.12]). From this result together with Theorem 14 we obtain the following theorem.

Theorem 26 For every $d \geq 2$ and $N \geq 2$ there exists a lattice point $\mathrm{g} \in \mathbb{Z}^{d-1}$ such that

$$
\operatorname{wce}\left(\mathcal{P}_{N}(\boldsymbol{g}), \stackrel{\circ}{\mathbf{B}}_{p, \theta}^{s}\right) \lesssim \frac{(\log N)^{(d-1)(s+1-1 / \theta)}}{N^{s}} .
$$

However, it remains an open question how to construct, for given $d$ and $N$, lattice points $\boldsymbol{g} \in \mathbb{Z}^{d-1}$ which achieve the lower bound (16). Without loss of generality one can restrict oneself to the search space $\{g \in\{1,2, \ldots, N-1\}: \operatorname{gcd}(g, N)=1\}^{d-1}$ of size $\varphi(N)^{d-1}$, where $\varphi$ denotes Euler's totient function. This is too large for a full search already for moderately large $N$ and $d \geq 3$. So far one relies on computer search to find good generating vectors, usually based on the fast component-by-component construction [30,31]. 
Korobov [32] suggested considering lattice point sets with generating vectors $\boldsymbol{g}=$ $\left(1, g, g^{2}, \ldots, g^{d-1}\right)$ in $\mathbb{Z}^{d}$ with $g \in\{1,2, \ldots, N-1\}$ such that $\operatorname{gcd}(g, N)=1$. The size of the search space for lattice points of this form reduces to $\varphi(N)$. At least for prime powers $N$ and in dimension $d=3$, there is an existence result of Larcher and Niederreiter [33] for $g=\left(1, g, g^{2}\right)$ with

$$
\rho_{3}(N, \boldsymbol{g}) \geq \frac{C N}{(\log N)^{2}} .
$$

For $d>3$ this is open.

Acknowledgements Open access funding provided by Austrian Science Fund (FWF).

Open Access This article is distributed under the terms of the Creative Commons Attribution 4.0 International License (http://creativecommons.org/licenses/by/4.0/), which permits unrestricted use, distribution, and reproduction in any medium, provided you give appropriate credit to the original author(s) and the source, provide a link to the Creative Commons license, and indicate if changes were made.

\section{References}

1. Frolov, K.K.: Upper error bounds for quadrature formulas on function classes. Dokl. Akad. Nauk SSSR 231, 818-821 (1976)

2. Ullrich, M.: On "Upper error bounds for quadrature formulas on function classes". In: Frolov, K.K., Cools, R., Nuyens, D. (eds.) Monte Carlo and Quasi-Monte Carlo Methods 2014, vol. 163, pp. 571-582. Springer, Heidelberg (2016)

3. Temlyakov, V.N.: Cubature formulas, discrepancy, and nonlinear approximation. J. Complex. 19, 352-391 (2003)

4. Dubinin, V.V.: Cubature formulae for Besov classes. Izvestiya Math. 61(2), 259-283 (1997)

5. Temlyakov, V.N.: Approximation of Periodic Functions. Computational mathematics and analysis series. Nova Science Publishers Inc, Commack (1993)

6. Ullrich, M., Ullrich, T.: The role of Frolov's cubature formula for functions with bounded mixed derivative. SIAM J. Numer. Anal. 54(2), 969-993 (2016)

7. Nguyen, V.K., Ullrich, M., Ullrich, T.: Change of variable in spaces of mixed smoothness and numerical integration of multivariate functions on the unit cube. arXiv:1511.02036, (2015)

8. Skriganov, M.M.: Constructions of uniform distributions in terms of geometry of numbers, (Russian) Algebra i Analiz, 6(3), 200-230, (1994); translation in St. Petersburg Math. J., 6(3), 635-664, (1995)

9. Vybíral, J.: Function spaces with dominating mixed smoothness. Diss. Math. 436, 3-73 (2006)

10. Rvachev, V.A.: Compactly supported solutions of functional-differential equations and their applications. Rus. Math. Surv. 45, 87-120 (1990)

11. Schmeisser, H.-J., Triebel, H.: Topics in Fourier Analysis and Function Space. Wiley, Chichester (1987)

12. Stein, E.M., Weiss, G.: Introduction to Fourier Analysis on Euclidean Spaces, Princeton Mathematical Series 32. Princeton University Press, Princeton (1971)

13. Niederreiter, H.: Random Number Generation and Quasi-Monte Carlo Methods. SIAM, Philadelphia (1992)

14. Sloan, I.H., Joe, S.: Lattice Methods for Multiple Integration. Oxford Science Publications, New York (1994)

15. Niederreiter, H.: Quasi-Monte Carlo methods and pseudo-random numbers. Bull. Amer. Math. Soc. 84, 957-1041 (1978)

16. Niederreiter, H., Sloan, I.H.: Lattice rules for multiple integration and discrepancy. Math. Comp. 54, 303-312 (1990)

17. Sloan, I.H., Kachoyan, P.J.: Lattice methods for multiple integration: theory, error analysis and examples. SIAM J. Numer. Anal. 24, 116-128 (1987)

18. Khinchin, A.Y.: Continued Fractions. Dover Publications Inc, Mineola (1997)

19. Badziahin, D.A.: On multiplicatively badly approximable numbers. Mathematika 59(1), 31-55 (2013)

20. Beck, J.: Probabilistic diophantine approximation. I. Kronecker Seq. Ann. Math. 140, 451-502 (1994)

21. Bakhvalov, N.S.: On the approximate calculation of multiple integrals. J. Complex. 31, 502-516 (2015) 
22. Schmidt, W.M.: Simultaneous approximation to algebraic numbers by rationals. Acta Math. 125, 189-201 (1970)

23. Zaremba, S.K.: Good lattice points, discrepancy, and numerical integration. Ann. Mat. Pura Appl. 73, 293-317 (1966)

24. Temlyakov, V.N.: Error estimates for Fibonacci quadrature formulas for classes of functions with a bounded mixed derivative, (Russian) Trudy Mat. Inst. Steklov., 200, 327-335, (1991); translation in Proc. Steklov Inst. Math. 2(200), 359-367, (1993)

25. Zaremba, S.K. (ed.).: La méthode des "bons treillis" pour le calcul des intégrales multiples, (French). In: Proceeding of Symposium on Applications of Number Theory to Numerical Analysis in University of Montreal, Montreal, pp. 39-119. Academic Press, New York (1972)

26. Niederreiter, H.: Dyadic fractions with small partial quotients. Monatsh. Math. 101, 309-315 (1986)

27. Bourgain, J., Kontorovich, A.: On Zaremba's conjecture. Ann. Math. 2(180), 137-196 (2014)

28. Huang, S.Y.: An improvement to Zaremba's conjecture. Geom. Funct. Anal. 25, 860-914 (2015)

29. Zaremba, S.K.: Good lattice points modulo composite numbers. Monatsh. Math. 78, 446-460 (1974)

30. Nuyens, D., Cools, R.: Fast algorithms for component-by-component construction of rank-1 lattice rules in shift-invariant reproducing kernel Hilbert spaces. Math. Comp. 75, 903-920 (2006)

31. Nuyens, D., Cools, R.: Fast component-by-component construction of rank-1 lattice rules with a nonprime number of points. J. Complex. 22, 4-28 (2006)

32. Korobov, N.M.: Properties and calculation of optimal coefficients. Dokl. Akad. Nauk SSSR 132, 10091012 (1960). (In Russian)

33. Larcher, G., Niederreiter, H.: Optimal coefficients modulo prime powers in the three-dimensional case. Ann. Mat. Pura Appl. 155, 299-315 (1989) 